\title{
Introduction to A Research Agenda for Knowledge Management and Analytics
}

\section{Introduction}

Knowledge management (KM) has evolved over the years from the early 1980s coining of its name. By now (in 2020), organizations shouldn't even be talking about "knowledge management" - they should already be doing it! Unfortunately, this hasn't been the case for a number of reasons, including KM envisioned as mostly a long-term versus short-term payoff, misunderstanding the benefits of knowledge sharing, not recognizing those who exhibit learning and knowledge sharing behaviors, and a host of other reasons.

Even though a number of journals focus on knowledge management, many organizations still use ad hoc processes to develop and implement KM into their organizations. To help standardize some of these KM processes, the International Organization for Standardization (ISO) knowledge management systems standard was developed in 2018 .

Specifically, the ISO 30401 indicates that top management shall establish a knowledge management policy that:

(a) is appropriate to the purpose of the organization;

(b) provides a framework and guiding principles for setting, reviewing and achieving knowledge management objectives;

(c) includes a commitment to satisfy applicable regulatory and other requirements;

(d) sets expectations for all workers with regard to use of the knowledge management system and the cultivation of a culture that values knowledge;

(e) includes a commitment to continual improvement of the knowledge management system;

(f) manages the balance between knowledge sharing and knowledge protection. (https://www.iso.org/standard/68683.html) 
In addition to process considerations, the people and cultural components of $\mathrm{KM}$ are often overlooked in favor of the technology piece. The adage that " 80 percent of $\mathrm{KM}$ is people/culture/process and $20 \%$ is technology" should ring true to many successful organizations who have implemented knowledge management.

As KM has evolved over the years, what needs to be considered for the next generation of KM? The key word is "synergy". Unfortunately, KM is fading a bit and needs to be strengthened by aligning KM with other emerging areas, such as:

- Analytics

- Intuition-based decision making

- Entrepreneurship and Innovation

- Organizational strategy

- Artificial intelligence (AI)/machine learning

- Augmented reality

- IoT (Internet of Things)

- Intelligence amplification (IA).

In particular, as big data and analytics continue to grow, there are some natural synergies with KM and analytics. For example, KM could play a key role in the management and governance of the use of big data/analytics in organizational settings.

As cognitive computing, $\mathrm{AI}$ and machine learning continue to develop, KM can also play a role. For example, DARPA (Defense Advanced Research Projects Agency in the US) has the Lifelong Learning Machines Program that seeks to develop learning systems that continuously improve with additional experience, and rapidly adapt to new conditions and dynamic environments. Here KM plays a key part. In some of the author's recent research on intuition-based decision making, we see that experiential learning is critical in developing intuitive awareness (Liebowitz, 2019; Liebowitz et al., 2019). Again, many of the principles of KM apply here too.

Some other possible synergies for KM and analytics include business process mining. In fact, there is a Business Rules Conference (mainly for industry) held in late Fall each year in the U.S.A. In addition, KM should be part of the strategic intelligence of an organization-that is, strategic intelligence being the intersection of KM, Business Intelligence (Analytics), and Competitive Intelligence) (Liebowitz, 2006). 
In looking ahead, the KM educators and practitioners need to do the following:

- Promote greater dialogue between KM and the various communities;

- KM educators/practitioners must be somewhat adept in applying analytics tools, techniques and methodologies, and the Data Analytics educators/ practitioners must also develop appropriate KM skills;

- Further investigate areas for collaboration (cognitive computing, executive decision making, IoT, Scientometrics, etc.).

\section{KM Lessons Learned}

Have a senior champion and align your KM strategy with your organizational strategies, goals and objectives

Similar to any organizational initiative, it is critical that a senior champion exists to provide the financial and moral support for the KM program to be successful. Not only is senior champion support essential but also aligning the KM strategy with the organizational strategies, goals and objectives is crucial. Without this proper alignment, it would be difficult to assess and evaluate how well the KM initiative (or program) is performing relative to the organization's KPIs (Key Performance Indicators).

Develop a well-designed KM implementation plan (people, process and technology)

A solid KM strategy and resulting KM implementation plan/roadmap should consist of People/Culture, Process, and Technology components. As previously mentioned, the $80-20$ rule applies where $80 \%$ deals with people/culture and process issues, and $20 \%$ is technology. Many organizations will build out a three-year KM implementation plan based on the KM projects proposed as part of the KM strategy.

Develop a formal knowledge retention strategy-start from day one of the employee's life with the organization

Organizational amnesia often occurs, especially as the tenure of employees in the organization increases over the years. With the "graying workforce," due to the demographics in society, knowledge retention should be an important part of the organization's human capital strategy. In fact, a knowledge retention formal process (perhaps an SOP-Standard Operating Procedure) should be created and used from day one of the employee's life with the organization. As 
the years go by, employees may forget some valuable knowledge and being able to capture this knowledge at the point of "knowledge existence" would reduce the risk of losing it. In addition, the knowledge-engineering paradox exists which states that the more experienced the expert, the more compiled the knowledge, and the harder it is to extract or elicit this knowledge. Thus, doing knowledge retention capture early on will alleviate this issue.

Incorporate KM as part of human capital strategy, succession planning, workforce development, strategic planning, and/or quality management

Many organizations don't need to have a separate KM Program per se. KM can be part of some of the existing programs in the organization. For example, NASA applies KM as part of their project management and risk management efforts. The FDA (Food and Drug Administration in the U.S.A.) has applied knowledge management as part of their quality management efforts. The Annie E. Casey Foundation in Baltimore has incorporated KM as part of their strategic planning efforts. Many other organizations apply KM as part of their human capital strategy, succession planning and workforce development.

Be thoughtful in your approach (knowledge audit, social network analysis, etc.)

In the spirit of knowledge sharing, the KM community can apply some existing techniques to baseline an organization in terms of its knowledge sharing practices and collaboration networks. A knowledge audit is often used to determine the KM practices, gaps and issues for developing a KM strategy. This approach is modeled after the information audit from the Information Systems community. In addition, the use of Social Network Analysis, borrowed from the sociology and education disciplines, can be adeptly used to map knowledge flows and gaps in organizations. Various knowledge management instruments have already been validated over the years which could provide value to the organization under study, so that the wheel isn't reinvented unnecessarily.

\section{Align your KM approaches to fit your organizational culture}

Typically, KM approaches are classified under codification ("collection") and personalization ("connection") techniques. Codification techniques could be lessons learned systems, yellow pages of expertise (although, "connection" applies here as well), online multimedia asset repositories, and the like. Personalization/connection techniques include mentoring programs, job shadowing, job rotation and others. Most of the time, organizations will apply 
both types of approaches but one type will be dominant. To help determine the dominant set of approaches (i.e., codification vs. personalization), organizations should determine if their employees are more systems-oriented or people-focused. The Myers-Briggs Type Indicator, for example, can be used to determine various dimensions of individuals, ranging from introvert/extrovert, sensing/intuitive, thinking/feeling and judging/perceiving. For example, NASA probably has more ISTJ types of individuals where a systems-orientation is preferred. In this case, NASA probably errs more on applying the codification approaches (such as the NASA Engineering Network/Lessons Learned Information System) versus personalization approaches (although, in NASA's case, both approaches are used; see APPEL—https://appel.nasa.gov).

\section{Celebrate the successes, then bring in the bittersweet stories}

Showing success through quick-win KM pilots is critical to further convince the naysayers. Celebrate the successes first and then bring in the failures or stories of things that didn't go right. Lessons learned are based on experience, whether positive or negative. Typically, people may learn more from failures than from successes; thus, both experiences are important to capture (Liebowitz, 2016).

\section{Develop KM metrics (especially outcome measures)}

Many organizations get caught up on system and output measures. For example, seeing how many times a website is accessed may not necessarily translate to innovations or other outcome measures. In academia, professors and administrators should be more interested in "learning outcomes" than "teaching outcomes." In the same way, KM initiatives should contribute towards outcome measures relating to the organization's KPIs and strategic goals.

Don't force-fit technology (people/culture/process are where the rubber hits the road)

An old adage is, "if all you know is a hammer, then every problem looks like a nail." Thus, KM professionals shouldn't force-fit technology to the KM-related problem. Look first at the problem requirements, then decide if there is an appropriate technology that could be used to address the issue. Again, the key elements are usually the people/culture/process in terms of gaining the most value from a KM initiative. 
$\mathrm{KM}$ is just one part of your "strategic intelligence"

Knowledge management should be one of the skill sets in one's decision making kit. It should be part of one's "strategic intelligence" (Liebowitz, 2006), whereby business intelligence/analytics, Artificial Intelligence (AI), competitive intelligence (CI), and other key disciplines play a major role.

\section{Knowledge Sharing Tenets for Success}

In order to be successful for KM programs, here are some knowledge sharing tenets that should be followed (Liebowitz, 2012):

- Enhance reward and recognition system to include learning and knowledge sharing competencies;

- Acquaint people with knowledge sharing and its benefits;

- Share the message that with creativity comes failure and we all benefit from talking about our successes and our failures;

- Integrate knowledge sharing into everyone's job;

- Educate people about what types of knowledge are valuable and how they can be used;

- Make sure the technology works for people, not vice versa.

\section{References}

Liebowitz, J. (ed.) (2006), Strategic Intelligence: Business Intelligence, Competitive Intelligence, and Knowledge Management, CRC Press.

Liebowitz, J. (ed.) (2012), The Knowledge Management Handbook: Collaboration and Social Networking, 2nd edition, CRC Press.

Liebowitz, J. (ed.) (2016), Successes and Failures of Knowledge Management, Morgan Kaufmann/Elsevier.

Liebowitz, J. (ed.) (2019), Developing Informed Intuition for Decision Making, Taylor $\&$ Francis.

Liebowitz, J., Y. Chan, T. Jenkins, D. Spicker, J. Paliszkiewicz and F. Babiloni (eds.) (2019), How Well Do Executives Trust Their Intuition?, Taylor \& Francis. 